Thorax, 1981, 36, 95-98

\title{
Auscultation of the heart since Laënnec
}

\author{
AUBREY LEATHAM
}

From St George's Hospital, London

With the advent of accurate phonocardiography in the early 1950s and with echocardiography in the last decade to give the precise timing of valve movement, auscultation of the heart is now on a firmly established basis.

In the pre-stethoscope era, perhaps the most perceptive comment was that of Robert Hooke to the Royal Society quoted by McKusick," "I have been able to hear very plainly the beating of a man's heart. . . . Who knows I say that it may be possible to discover the motions of the internal organs. ..."

Laënnec's great contribution to medicine was the invention of the stethoscope in 1819. His main interest, however, was auscultation of the lungs rather than the heart. While he correctly timed the first heart sound to coincide with contraction of the ventricle, unfortunately he attributed the second sound to atrial contraction and was thus unable to make accurate correlations between murmurs and pathological findings. After Hope's animal experiments which gave the timing of the first and second sounds (1832), rapid advances were made. By the end of the century nearly every notion in use today had been described, including inspiratory splitting of the second sound by Potain in 1866, but the accurate theories were outnumbered by the innacurate, and proof of the origin of sounds and their significance could not be obtained until relatively recent times.

\section{Heart sounds}

Heart sounds are divisible into relatively loud high-frequency sounds, thought by Dock nearly 50 years ago to be caused by the terminal halt of opening and closing valves, and the low frequency, soft (to the ear) ventricular filling sounds (third and atrial) which will not be discussed further here since no new light has been thrown on their mechanism.

Splitting of the first heart sound into two highfrequency components can be heard over the lower end of the sternum in most normal subjects

Address for reprint requests: Dr Aubrey Leatham, St Georges' Hospital, Blackshaw Road, London SW17 0QT. when using a rigid diaphragm stethoscope, but the identity of the two components has been the subject of debate for the last 20 years. ${ }^{2-8}$ Initially, it was assumed that splitting indicated asynchrony of atrioventricular valve closure at the onset of ventricular systole, with the mitral component before the tricuspid as suggested by the site of maximum intensity and the relation to the carotid pulse. $^{2}$ Further support for this was the finding of increased delay of the second component in most patients with right bundle branch block with ectopic beats arising from the left ventricle, and with pacing from the left ventricle. ${ }^{3}$ With intracardiac phonocardiography and animal experiments, however, the sound attributed to tricuspid valve closure could not be identified, and there was a strong school of thought in the United States which denied the existence of vibrations originating in the right side of the heart. ${ }^{4}$ The situation has at least been clarified using high speed, high definition echocardiograms simultaneously with phonocardiograms. It has now been shown that the first high frequency component of the first sound is exactly synchronous with the final halt of the closing mitral valve, and the second component, when separately identifiable, is exactly synchronous with that of the tricuspid valve. ${ }^{5-7}$ The interval between the components (typically $30 \mathrm{~ms}$ ) is greater than expected from the slight asynchrony of ventricular contraction and we have had to wait until very recently for a reasonable explanation of this phenomenon. It has now been shown that the precise timing of mitral valve closure is closely dependent on the P-R interval. ${ }^{8}$ Echocardiography has confirmed that atrial contraction and relaxation opens and then closes the atrioventricular valves. With a P-R interval of $0.16 \mathrm{~s}$ or more, left atrial contraction opening the mitral valve in pre-systole, also has time to close it almost completely. Thus the final half of closure occurs very soon after the onset of the left ventricular pressure pulse. Right atrial contraction has much less effect on the timing of tricuspid closure, presumably because right atrial contraction is less effective, and ejection is into a more flaccid ventricle. Thus 
the tricuspid valve is more fully open than the mitral at the onset of ventricular systole and therefore takes longer to be closed. The difference in timing between mitral and tricuspid closure, and thus the corresponding sounds, therefore tends to be greater than the degree of asynchrony of the corresponding ventricular pressure pulses. Thus physiological splitting of the first sound may be surprisingly wide and the tricuspid closure sound is sometimes difficult to differentiate from an ejection sound.

Echo observations on the timing of mitral valve closure have also thrown light on the variation in intensity of the first heart sound, which has been known for many years to be influenced by the P-R interval. ${ }^{9-11}$ While echocardiography confirmed the relation between intensity of the first sound and the position of the mitral cusps at the moment of ventricular contraction, it seemed strange that varying distance of travel of such flimsy structures should cause such great alterations in intensity of sound produced by their final closing halt and it was suggested that late closure as in mitral stenosis was responsible for the loud sounds. ${ }^{12}$ It has now been shown that the wide open valve associated with a short P-R interval, having a greater distance to travel, does indeed close late. ${ }^{13}$ Mitral closure is completed at a time when the rate of increase of ventricular pressure is much greater, and so the valve closes with high velocity and creates a loud sound. When the P-R interval is long, however, atrial contraction has had time almost to appose the mitral leaflets, and final closure will occur earlier when the rate of pressure change is minimal, producing only a soft sound. In this way, echocardiography has been invaluable for gaining an understanding of the principles governing intensity and splitting of the first heart sound in normal subjects.

Following on from these observations, studies have been made of the time interval between mitral and tricuspid closure and pulmonary valve opening in right bundle branch block and in pulmonary hypertension. It seems possible to distinguish proximal right bundle branch block where the onset of ventricular contraction and tricuspid closure are late, from distal or arborisation block where the onset of ventricular contraction is not delayed but activation of the right ventricle is slow ${ }^{14}$; in both varieties the pulmonary valve opens late. Proximal block is the usual explanation of isolated right bundle branch block with good prognosis found as a result of routine electrocardiographic examination and can be suspected by hearing wide splitting of the first sound. Distal block is usually associated with $\frac{\vec{F}}{\stackrel{F}{(}}$ more serious disease of the conducting tissue or of the myocardium. It can be suspected when split- $\frac{O}{\omega}$ ting of the first sound cannot be detected, despite electrocardiographic evidence of right bundle $\stackrel{\mathbb{\Omega}}{\Omega}$ branch block. With pulmonary hypertension the is isovolumic time of the right ventricle is usually $\overrightarrow{0}$ prolonged and the late opening of the pulmonary valve can be detected by echo if a late ejection $\vec{\omega}$ sound is not audible. ${ }^{15}$ This is a useful addition to $\vec{F}$ the other clinical and non-invasive signs of pul- $\vec{x}$ monary hypertension, namely a big A wave in the of venous pulse, a sustained para-sternal heave, an abnormally loud pulmonary component of the $O$ second sound in relation to the preceding aortic 의 component, transmission of the pulmonary com- ponent to the apex, and the usual electrocardio- T⿱乛龰 graphic and radiological evidence. Thus, $\frac{0}{\mathrm{C}}$ pulmonary hypertension should seldom be a "catheter diagnosis" only.

Aortic ejection sounds are caused by abnormal $\vec{\oplus}$ aortic cusps which remain mobile but cannot fold $\stackrel{\infty}{\cdot}$ neatly against the aortic wall during ejection; they can be identified precisely by echocardiography since they coincide with the final halt of the opening valve cusps. Thus a haemodynamically normal bicuspid aortic valve-the most common $\stackrel{\unrhd}{\unrhd}$ congenital anomaly of the heart-can be identi- $\overrightarrow{\vec{F}}$ fied. This is of interest since long-term follow-up $\frac{0}{3}$ discloses a small risk of bacterial endocarditis and the variable development of calcific aortic stenosis in later life. ${ }^{16} 17$ An aortic ejection sound is almost invariably obvious with aortic valve stenosis unless there is gross calcification and is invariably absent $\underset{x}{x}$ with subaortic obstruction unless there is an ad- $\dot{0}$ ditional bicuspid valve. Identification of an aortic ejection sound is also useful in deciding the aetiology of aortic regurgitation, since it is almost always present with a bicuspid regurgitant valve, 옥 is occasionally present with a rheumatic regurgi- $\rightarrow$ tant valve, but is absent when root dilatation is the cause of the aortic regurgitation. ${ }^{18}$

The components of the second heart sound have or been easier to identify and to understand, and $\mathcal{N}$ echocardiography has added little to our knowl- $\omega$ edge except for its ability to identify pulmonary으 closure in some cases when this is difficult one auscultation, or occasionally even on a phono- $\mathbb{\Phi}$ cardiogram. The major value of an understanding? of the second heart sound and its respiratory $\frac{T}{2}$ variations is in the examination of children, in $\frac{\vec{D}}{\mathbb{D}}$ whom "innocent" or physiological ejection systolic $\stackrel{\odot}{\mathbb{P}}$ murmurs are frequently heard. Physiological split- $\stackrel{\mathbb{Q}}{\varrho}$ ting almost excludes other causes of pulmonary? ejection murmurs such as atrial septal defect and $\delta$ 
pulmonary stenosis. Moreover, the width of splitting (delay of P2) in pulmonary stenosis gives an assessment of severity of obstruction which is almost as accurate as measurement of the pressure gradient across the pulmonary valve. ${ }^{19}$

Identification of a mitral opening snap is usually easy, particularly if a high frequency sound can be heard or recorded in addition to the aortic and pulmonary components of the second heart sound, but in difficult cases it can be identified by its coincidence with the opening halt of the mitral valve on echo. With calcific mitral stenosis, particularly when complicated by severe pulmonary hypertension, abnormal sounds and murmurs may be absent, and echocardiography is needed. It is also useful when the diagnosis of mitral stenosis is difficult as in the presence of aortic stenosis and, of course, it has proved far and away the most sensitive method for diagnosing myxoma when auscultatory signs are non-specific.

\section{Heart murmurs}

The division of heart murmurs into pansystolic regurgitant murmurs and mid-systolic ejection murmurs seems to have stood the test of time, provided that the murmur is analysed in relation to the relevant component of the second sound. ${ }^{20}$ For example, the pan-systolic murmur of mitral regurgitation engulfs A2 but stops before P2; if splitting is wide because of shortening of left ventricular systole, this may give a false impression of an ejection murmur. In severe mitral regurgitation there is a great shortening of left ventricular ejection, A2 may be very early, and occasionally an echo is needed for its identification. A phonocardiogram with a synchronous carotid pulse is often useful for analysing an obscure apical systolic murmur to get its relationship to the components of the second sound. It is particularly useful in the diagnosis of hypertrophic cardiomyopathy with a systolic murmur from obstruction to left ventricular outflow which tends to continue nearly to the aortic component of the second heart sound, but there is a gap shown on the phonocardiogram between the end of the murmur and A2. Differentiation between aortic valve and infundibular obstruction can usually be made by noting the presence or absence of an ejection sound. Echocardiography usually can confirm the site of the obstruction and, in experienced hands, gives some indication of the degree of calcification, if any. Thus the cause of an obscure outflow systolic murmur can be elucidated without the need to resort to cardiac catheterisation, although there is at present no good non-invasive method for determining severity.

There has been an improvement in our understanding of diastolic murmurs with the advent of echocardiography. ${ }^{21}$ Ventricular filling murmurs without atrioventricular valve obstruction are related to the semi-closing of the valve while forward flow is still continuing, the best example being the short mitral diastolic murmur in severe mitral regurgitation. Thus Austin Flint's observations on the mechanism of the mitral diastolic murmur in pure aortic regurgitation have been confirmed. ${ }^{22}$

In conclusion, Laënnec with his interest in diagnosis would have been gratified to know that with careful history taking, followed by physical examination of the cardiovascular system, a correct diagnosis can be made in the majority of cases. Indeed with the additional aid of an electrocardiogram, a radiograph of the chest, and an echocardiogram, complicated invasive investigations are seldom necessary except in neonatal diagnosis and as a prelude to cardiac surgery.

\section{References}

1 McKusick VA. Cardiovascular sound. London: Baillière Tindall and Cox, 1958.

2 Leatham A. Splitting of the first and second heart sounds. Lancet 1954; 2:607.

3 Haber E, Leatham A. Splitting of heart sounds from ventricular asynchrony in bundle branch block, ventricular ectopic beats, and artificial pacing. Br Heart J 1965; 27:691.

4 Luisada A. "Tricuspid" component of the first heart sound. In Monograph No 46 of Am Heart Assoc, 1975.

5 Crews $T$, Pridie $R$, Benham $R$, Leatham A. Auscultatory and phonocardiographic findings in Ebstein's anomaly: correlation of first heart sound with ultrasonic recordings of tricuspid valve movement. Br Heart J 1972; 34:681.

6 Leatham A, Leech $G$. Observations on the relationship between heart sounds and valve movements by simultaneous echo and phonocardiography. Br Heart J 1975 (abstr); 37:557.

7 Waider W, Craige E. First heart sound and ejection sounds: echocardiographic and phonocardiographic correlation with valvular events. Am J Cardiol 1975; 35:3.

8 Brooks N, Leech G, Leatham A. Factors responsible for normal splitting of first heart sound. High speed echophonocardiographic study of valve movement. Br Heart J 1979; 42:695.

9 Wolferth C, Margolies A. Certain effects of auricular systole and the prematurity of beat on the intensity of the first heart sound. Trans Assoc 
Am Phys 1930; 45:44.

10 Levine S, Harvey W. Clinical auscultation of the heart. Philadelphia: WB Saunders, 1949.

11 Burgraaf $\mathrm{GW}$, Craige E. The first heart sound in complete heart block: phonechocardiographic correlations. Circulation 1974; 50:17.

12 Leatham A. Auscultation of the heart and phonocardiography. Second edition. London: Churchill Livingston, 1975.

13 Leech G, Brooks N, Green-Wilkinson A, Leatham A. Mechanism of influence of P-R interval on loudness of first heart sound. Br Heart J 1980; 43:138.

14 Brooks N, Leech G, Leatham A. Complete right bundle branch block. Echophonocardiographic study of first heart sound and right ventricular contraction times. Br Heart J 1979; 41:637.

15 Mills P, Leech G, Leatham A, Ginks W. Noninvasive estimation of pulmonary artery enddiastolic pressure. Circulation 1975; 5152 suppl II:192.
16 Leech G, Mills P. Leatham A. The diagnosis of $\stackrel{\vec{F}}{?}$ a non-stenotic bicuspid aortic valve. Br Heart $J$ 을 1978; 40:941.

17 Mills P, Leech G, Davies M, Leatham A. The $\frac{\bar{\rho}}{\frac{5}{\sigma}}$ natural history of a non-stenotic bicuspid aortic $\unrhd$ valve. Br Heart J 1978; 40:851.

18 Leech GJ, Davies MJ, Brooksby I, Parker o Braimbridge MV. The use of M-mode echocardi-. ography to determine the pathogenesis of aortic $\vec{A}$ regurgitation. Br Heart J 1978; 40:460.

19 Leatham A, Weitzman D. Auscultatory and $\overrightarrow{\vec{z}}$ phonocardiographic signs of pulmonary stenosis. Br Heart J 1957; 19:303.

20 Leatham A. Auscultation of the heart: Goulstonian lecture. Lancet 1958; 1:757.

21 Fortuin NJ, Craige E. Echocardiographic studies $\mathrm{O}$ of genesis of mitral diastolic murmurs. $\mathrm{Br} \mathrm{Heart}-$ J 1973; 35:75.

22 Fortuin NJ, Craige E. On the mechanism of the Austin Flint murmur. Circulation 1972; 45:558. 